\title{
Global miRNA expression analysis of serous and clear cell ovarian carcinomas identifies differentially expressed miRNAs including miR-200c-3p as a prognostic marker
}

Bente Vilming Elgaaen ${ }^{1 *}$, Ole Kristoffer Olstad ${ }^{2}$, Kari Bente Foss Haug ${ }^{2}$, Berit Brusletto ${ }^{2}$, Leiv Sandvik ${ }^{3,4}$, Anne Cathrine Staff ${ }^{3,5}$, Kaare M Gautvik ${ }^{2,3}$ and Ben Davidson ${ }^{3,6}$

\begin{abstract}
Background: Improved insight into the molecular characteristics of the different ovarian cancer subgroups is needed for developing a more individualized and optimized treatment regimen. The aim of this study was to a) identify differentially expressed miRNAs in high-grade serous ovarian carcinoma (HGSC), clear cell ovarian carcinoma (CCC) and ovarian surface epithelium (OSE), b) evaluate selected miRNAs for association with clinical parameters including survival and c) map miRNA-mRNA interactions.

Methods: Differences in miRNA expression between HGSC, CCC and OSE were analyzed by global miRNA expression profiling (Affymetrix GeneChip miRNA 2.0 Arrays, $n=12,9$ and 9, respectively), validated by RT-qPCR ( $n=35,19$ and 9, respectively), and evaluated for associations with clinical parameters. For HGSC, differentially expressed miRNAs were linked to differentially expressed mRNAs identified previously.

Results: Differentially expressed miRNAs $(n=78)$ between HGSC, CCC and OSE were identified (FDR $<0.01 \%)$, of which 18 were validated $(p<0.01)$ using RT-qPCR in an extended cohort. Compared with OSE, miR-205-5p was the most overexpressed miRNA in HGSC. miR-200 family members and miR-182-5p were the most overexpressed in HGSC and CCC compared with OSE, whereas miR-383 was the most underexpressed. miR-205-5p and miR-200 members target epithelial-mesenchymal transition (EMT) regulators, apparently being important in tumor progression. miR-509-3-5p, miR-509-5p, miR-509-3p and miR-510 were among the strongest differentiators between HGSC and CCC, all being significantly overexpressed in CCC compared with HGSC. High miR-200c-3p expression was associated with poor progression-free $(p=0.031)$ and overall $(p=0.026)$ survival in HGSC patients. Interacting miRNA and mRNA targets, including those of a TP53-related pathway presented previously, were identified in HGSC.

Conclusions: Several miRNAs differentially expressed between HGSC, CCC and OSE have been identified, suggesting a carcinogenetic role for these miRNAs. miR-200 family members, targeting EMT drivers, were mostly overexpressed in both subgroups, among which miR-200c-3p was associated with survival in HGSC patients. A set of miRNAs differentiates CCC from HGSC, of which miR-509-3-5p and miR-509-5p are the strongest classifiers. Several interactions between miRNAs and mRNAs in HGSC were mapped.
\end{abstract}

Keywords: Ovarian carcinoma, MicroRNA, Microarray, Quantitative PCR, Survival

\footnotetext{
* Correspondence: bente.vilming.elgaaen@medisin.uio.no

'Department of Gynecological Oncology, Oslo University Hospital (OUH), The

Norwegian Radium Hospital, Postbox 4953 Nydalen 0424, Oslo, Norway

Full list of author information is available at the end of the article
} 


\section{Background}

Ovarian cancer is the fourth and fifth most frequent cause of cancer death in women in Norway and the U.S., respectively $[1,2]$. Two-thirds of patients have advancedstage disease (International Federation of Gynecology and Obstetrics [FIGO] stage III-IV) at diagnosis, resulting in 5-year survival at $<30 \%[1,2]$.

Ovarian carcinoma (OC) constitutes about $90 \%$ of ovarian cancers, and is a heterogeneous group of tumors, encompassing several distinct subgroups with respect to molecular profiles, biological behavior and clinical features [3-6]. Nevertheless, OC patients generally receive similar, non-individualized treatment. Therefore, improved insight into the molecular characteristics of the different OC subgroups may aid in development of a more subgroup-specific treatment, thereby improving prognosis.

microRNAs (miRNAs) are short, non-coding RNA molecules, which by targeting mRNAs cause mRNA degradation or translational repression [7]. Since a single miRNA may have multiple different mRNA targets and conversely, a given mRNA might be targeted by multiple miRNAs, miRNAs play a central role in regulating gene expression. Alterations in miRNA expression level may consequently alter the level of a wide spectrum of mRNAs and subsequently cellular functions.

miRNAs show abnormal expression patterns in different cancer forms [8]. Some act as tumor suppressor genes or oncogenes and may therefore be important in cancer development $[9,10]$. Various gene expression analysis approaches, including microarrays, have identified aberrantly expressed miRNAs in OC $[9,11-26]$, of which some are related to progression [13], outcome [18-24] and chemotherapy resistance [23-25]. However, the studies have in general utilized non-subgroup specific tumors [11], and only a few included normal ovarian surface epithelium (OSE) [15-17], which has been shown to be valid control material $[27,28]$.

The aim of this study was to identify miRNAs differentially expressed between moderately and poorly differentiated serous $\mathrm{OC}$, referred to as high-grade serous OC (HGSC), clear cell OC (CCC) and scrapings from OSE, and to evaluate their association with clinical parameters, including survival. To identify potential key molecular pathways of the carcinogenesis of HGSC, differentially expressed miRNAs and mRNAs identified previously [29] were linked. We have identified several miRNAs differentially expressed between HGSC, CCC and OSE, including miR-200c-3p with apparent clinical relevance in HGSC. Several interactions of potential oncogenic function between aberrantly expressed miRNAs and mRNAs in HGSC have also been mapped, including interactions between miR-200 members and the epithelial-mesenchymal transition (EMT) regulators ZEB1 and ZEB2.

\section{Methods}

\section{Ethics statement}

The study was approved by the Regional Committee of Medical and Health Research Ethics of South-Eastern Norway (ref.no.530-02163 and S-04300) and all participants signed informed consent.

\section{Patients and material}

Women were enrolled prior to operations for gynecological diseases at Oslo University Hospital (OUH) during 20032012. Patient information was obtained from hospital records and preoperative interviews. Patients were evaluated routinely [29] and follow-up data, including clinical examinations, laboratory analyses and imaging were available for all patients. CA125 level was used as marker for therapy response. CA125 normalization ( $<35 \mathrm{kU} / \mathrm{L})$ was defined as optimal when achieved within four cycles of chemotherapy. Time until progression and time until death were defined as the time interval from the date of surgery to the date of first confirmed disease recurrence and to the date of death, respectively. Disease progression was based on CA125 level increase according to GCIG (Gynecologic Cancer Intergroup) criteria (www.gcig.gcs.org) and verified clinical relapse, and the date of first event was used. Clinical data were current as of March 20, 2013.

Tumors comprised primary OC obtained pre-chemotherapy. OSE samples were collected from patients with benign diseases, as previously described [27]. Tumors were snap-frozen in liquid nitrogen immediately after harvesting, whereas OSE samples were transferred to QiaZol solution (Invitrogen, Carlsbad, CA). All samples were stored at $-80^{\circ} \mathrm{C}$ until processed.

The histological classification and clinical staging were according to the World Health Organization classification and FIGO, respectively. Tumors were reviewed by a gynecological pathologist (BD) to confirm the histological type and grade. A frozen section from all biopsies was examined prior to RNA isolation to ensure a tumor component of at least $50 \%$ and absence of necrosis.

\section{RNA preparation}

Frozen tumors $(<50 \mathrm{mg})$ were homogenized directly for 3 minutes in $700 \mu$ l QIAzol using a TissueLyzer (Qiagen, Hilden, Germany). Total RNA was extracted using the miRNeasy Mini Kit (Qiagen) and Phase Lock Gel ${ }^{\mathrm{mx}}$ Heavy (5 PRIME GmbH, Hamburg, Germany). RNA was quantified with a NanoDrop ${ }^{\circ}$ ND-1000 Spectrophotometer (Saveen Werner, Malmö, Sweden), and quality assessed on Agilent 2100 Bioanalyzer RNA 6000 Nano Kits (Agilent Technologies, Palo Alto, CA). All samples showed adequate RNA quantity and quality.

\section{Global miRNA expression profiling}

Global miRNA expression was analyzed in 12 HGSC, 9 CCC and 9 OSE samples. Total RNA (400 ng) was used 
for biotin labeling of miRNA by the Genisphere FlashTag HSR kit following the manufacturer's recommendations (Genisphere, Hatfield, PA). Labeled miRNAs were hybridized to the GeneChip miRNA 2.0 Array (Affymetrix, Santa Clara, CA), representing 1,105 mature human miRNAs, as recommended by the manufacturer. Arrays were washed and stained using the FS-450 fluidics station (Affymetrix). Signal intensities were detected by Hewlett Packard Gene Array Scanner 3000 7G (Hewlett Packard, Palo Alto, CA). Microarray data were deposited in NCBI's Gene Expression Omnibus (GEO) [30] and are accessible through GEO Series accession number GSE47841 (http://www.ncbi.nlm.nih.gov/geo/query/acc. cgi?acc=GSE47841).

\section{Quantitative reverse transcription-polymerase chain reaction (RT-qPCR)}

Selected candidate miRNAs were validated by RT-qPCR in all samples analyzed by global miRNA expression profiling (except one excluded) and in additional samples, totaling 35 HGSC, 19 CCC and 9 OSE samples. Custommade TaqMan Low Density Array (TLDA) cards for human miRNA expression analysis (Applied Biosystems, Life Technologies, Carlsbad, CA) were used for quantification of specific miRNAs, each card allowing 384 simultaneous qPCR reactions of 24 different miRNAs run in duplicates. Included were two selected reference genes and one mandatory control (U6 (mammu6) snRNA).

Total RNA (350 ng) was applied for reverse transcription (RT) with stem-looped RT primer-mix, enabling synthesis of cDNA from mature miRNAs. Unbiased custom-based pre amplification was performed according to protocols, using gene-specific forward and reverse primers. The PCR reactions were performed on Unocycler (VWR International, B-3001 Leuven, Belgium). The TLDA cards were used for further PCR-amplification on a ViiA7 ${ }^{\text {tw }}$ Real Time PCR system thermocycler and analyzed with ViiA7 RUO Software (Applied Biosystems, Life Technologies).

Relative gene expression levels were calculated using the comparative crossing threshold method of relative quantification ( $\Delta \Delta \mathrm{Cq}$ method) [31,32], and presented as relative quantification cycle $(\Delta \mathrm{Cq})$ and fold change (FC) values. $\Delta \mathrm{Cq}$ was designated as the mean $\mathrm{Cq}$ (mean of duplicates) of a miRNA in a sample subtracted by the mean Cq (mean of duplicates) of two reference genes in the same sample. Based on recommendations from the manufacturer and comparison between the microarray and RT-qPCR analyses, Cq expression cutoff was set to 30 , which was applied for calculations. For analyzing associations with clinical parameters, $\Delta \Delta \mathrm{Cq}$ was calculated as mean $\Delta \mathrm{Cq}$ of the OSE controls subtracted by $\Delta \mathrm{Cq}$ of each tumor sample. For comparison of mean expression levels between different groups, $\Delta \Delta \mathrm{Cq}$ was calculated as mean $\Delta \mathrm{Cq}$ of one group subtracted by mean $\Delta \mathrm{Cq}$ of another group $\left(\Delta \mathrm{Cq}_{\mathrm{OSE}}-\Delta \mathrm{Cq}_{\mathrm{HGSC}}, \Delta \mathrm{Cq}\right.$ OSE $-\Delta \mathrm{Cq}_{\mathrm{CCC}}$; $\left.\Delta C q_{\mathrm{HGSC}}-\Delta \mathrm{Cq} \mathrm{q}_{\mathrm{CCC}}\right)$. FC was designated as $2^{\Delta \Delta \mathrm{Cq}}$.

All miRNAs analyzed were from Homo sapiens (hsa) and the prefix hsa was therefore excluded.

\section{Ingenuity pathway analysis (IPA)}

Data were analyzed through the use of IPA (Ingenuity ${ }^{\circ}$ Systems, www.ingenuity.com), which was used for identifying biological functions and related diseases and for mapping mRNA-miRNA interactions.

\section{Statistical analysis}

For computational analysis of the microarray data, scanned images were processed using the AGCC (Affymetrix GeneChip Command Console) software, and the CEL files were imported into Partek Genomics Suite software (PGS; Partek, Inc., St Louis, MO). The Robust Multichip Analysis (RMA) algorithm was applied for generation of relative signal values and normalization. For expression comparisons of different groups, a 1-way ANOVA model followed by calculation of FDR was used. Results were expressed as FC and p-values. Signal values were subjected to a non-supervised cluster analysis using the Euclidean/ average linkage algorithm.

Associations between signal values and progression-free survival (PFS) and overall survival (OS) were evaluated by Cox regression analyses followed by FDR correction. FDR q-values of 0.1 and 0.25 were used as significance levels for PFS and OS, respectively.

When comparing $\Delta \mathrm{Cq}$ values in different histological subgroups, a two-sided independent samples t-test was used since the $\Delta \mathrm{Cq}$ values were close-to-normally distributed. Associations between FC values of the RT-qPCR analyses and clinical parameters were evaluated. In order to decide whether expression of a miRNA was significantly associated with PFS and OS, Cox regression analyses were used. When significant, Kaplan-Meier plots were used to estimate survival curves for tertiles of the expression variable. To compare miRNA expression levels in two groups of patients, a two-sided Mann-Whitney U-test was used, since the FC expression levels were not normally distributed. The results for each group are presented as medians.

A significance level of $1 \%$ was used for differential miRNA expression, and 5\% when analyzing associations with clinical parameters. Statistical analyses were performed using the SPSS-PC package (Version 20, Chicago IL).

\section{Results}

\section{Patient characteristics}

Clinicopathologic data for the RT-qPCR cohort are shown in Table 1. All HGSC patients were diagnosed with FIGO stage IIIc/IV, whereas CCC patients were diagnosed at all stages due to limited patient material. The 
Table 1 Clinicopathological and laboratory information for patients selected for RT-qPCR analysis

\begin{tabular}{|c|c|c|c|}
\hline \multicolumn{2}{|l|}{ Parameter } & $\mathrm{HGSC}^{a}, \mathrm{n}=35$ & $\mathrm{CCC}^{a}, \mathrm{n}=19$ \\
\hline \multicolumn{2}{|l|}{ Age; mean \pm SD (range) } & $64.0 \pm 11.3(45-87)$ & $63.9 \pm 15.3(28-83)$ \\
\hline \multicolumn{2}{|c|}{ Preoperative CA125 (kU/L); mean \pm SD } & $3023 \pm 4129$ & $1438 \pm 2198$ \\
\hline \multirow[t]{4}{*}{ FIGO stage } & I & 0 & 10 \\
\hline & $\|$ & 0 & 3 \\
\hline & III & 25 & 5 \\
\hline & IV & 10 & 1 \\
\hline \multirow[t]{3}{*}{ Residual disease } & $0 \mathrm{~cm}$ & 3 & 13 \\
\hline & $<2 \mathrm{~cm}$ & 9 & 4 \\
\hline & $>2 \mathrm{~cm}$ & 23 & 2 \\
\hline \multicolumn{2}{|c|}{ Start of chemotherapy (days after surgery); mean \pm SD } & $27.7 \pm 11.6$ & $25.7 \pm 13.8$ \\
\hline \multirow[t]{2}{*}{ CA125 response ${ }^{b}$} & Yes & 31 & 15 \\
\hline & $\overline{\mathrm{No}}$ & 1 & 0 \\
\hline \multirow[t]{2}{*}{ Optimal CA125 normalization ${ }^{b}$} & Yes & 20 & 13 \\
\hline & No & 14 & 3 \\
\hline \multicolumn{2}{|c|}{ Median time (months) until progression (95\% Cl) } & $10(7-13)$ & $N A^{c}$ \\
\hline \multicolumn{2}{|c|}{ Median time (months) until death $(95 \% \mathrm{Cl})$} & $26(18-34)$ & $105(35-175)$ \\
\hline \multirow[t]{4}{*}{ Status at last follow-up ${ }^{d}$} & NED & 1 & 11 \\
\hline & AWD & 1 & 1 \\
\hline & $\mathrm{DOD}$ & 33 & 6 \\
\hline & DOUC & 0 & 1 \\
\hline
\end{tabular}

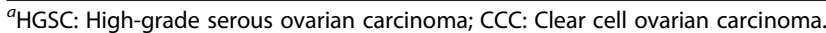

${ }^{b}$ According to the GCIG criteria (www.gcig.igcs.org). For HGSC: Two patients who received no postoperative treatment due to poor general condition, one patient with preoperative CA125 < 70; For CCC: One patient who received no postoperative treatment due to poor condition, three patients with preoperative CA $125<70$. ${ }^{c}$ Could not be calculated since the Kaplan-Meier survival curve stays above $50 \%$.

${ }^{d}$ NED $=$ No evidence of disease; AWD = Alive with disease; DOD = Dead of disease; DOUC = Dead of unrelated cause.

patients had no disease other than OC influencing survival, were Caucasian, and except for 1 with HGSC and 2 with CCC all were in good preoperative condition [27].

Primary surgery was performed in all patients. With the exception of 4 HGSC and 5 CCC patients, all received platinum-based chemotherapy. The 4 HGSC patients were considered to be in too poor general condition to tolerate chemotherapy. Among CCC patients, 1 received paclitaxelbased treatment, 1 was in too poor general condition, and 3 did not receive chemotherapy due to FIGO stage IA.

\section{Global miRNA expression analyses}

Seventy-eight miRNAs were differentially expressed between HGSC, CCC and OSE applying a FDR $<0.01 \%$. Complete p- and FC values are available in Additional file 1. Principal component analysis showed that these miRNAs could distinguish the 3 groups almost perfectly (Figure 1). Cluster analysis, visualized by a heatmap (Figure 2) showed almost perfect segregation of the 3 groups. Striking differences were observed between HGSC and OSE samples, whereas CCC had an intermediate profile. Moreover, miR-508-5p, miR-509-3p, miR-509-5p, miR-509-3-5p, miR-510 and miR-514b-5p clearly distinguished HGSC from CCC. OSE control samples were homogeneous.

\section{Evaluation of associations between global miRNA expression and survival}

Associations between miRNAs with signal values $>7$ $(\mathrm{n}=297)$ and PFS (FDR $\mathrm{q}<0.1)$ and OS (FDR $\mathrm{q}<0.25)$ were separately evaluated in HGSC and CCC. No statistically significant associations were found. However, when not corrected for multiple testing, 11 miRNAs had $\mathrm{p}<0.05$, indicating an association with survival. Of these, miR-505-5p, miR-1281 and miR-29b-2-5p had the lowest $\mathrm{p}$-values $(\mathrm{p}<0.03)$, all with potential association with survival in HGSC. These miRNAs were among the miRNAs chosen for RT-qPCR validation and subsequent evaluation for association with outcome in the extended patient cohort. Noteworthy, only miR-29b-2-5p was among the differentially expressed miRNAs shown in Figure 2.

\section{RT-qPCR validation of selected miRNAs}

Twenty-one miRNAs and 2 reference genes were selected for RT-qPCR validation in the extended patient material. Of these, 18 miRNAs (Table 2) were predominantly selected based on differential expression (Figure 2). All miRNAs with $\mathrm{FC}> \pm 20(\mathrm{n}=16)$ and 2 of the mRNAs with $\mathrm{FC}> \pm 15$ were included, reaching a highest FC 


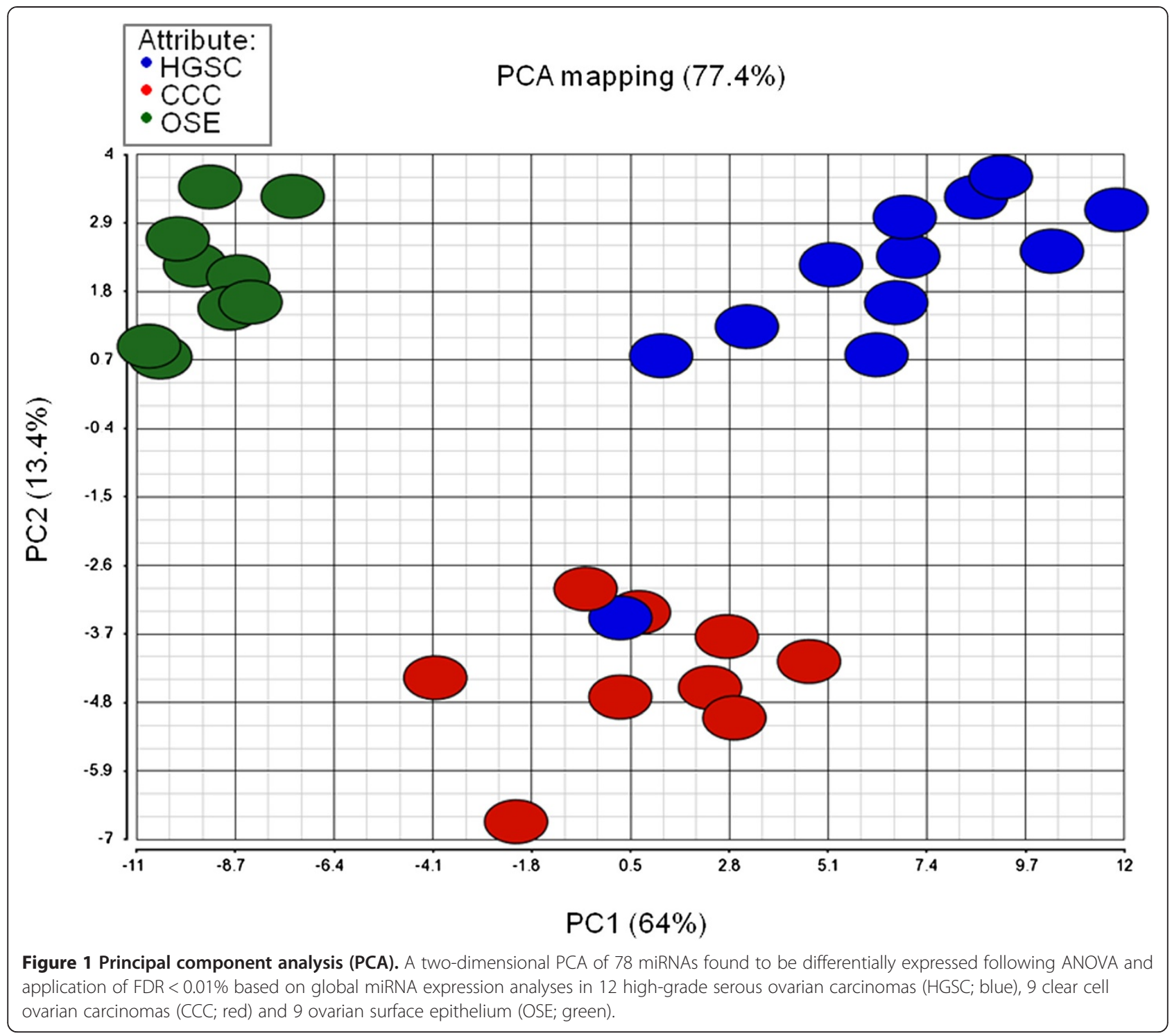

value of 105 . Additionally, the 3 above-mentioned miRNAs were selected based on possible association with survival. miR-24 and miR-26a were selected as reference genes, having the lowest expression variation (0.11 and 0.10 , respectively) in the global miRNA analysis. Their mean value reduced the variation to 0.029 , and their mean $\mathrm{Cq}$ value was therefore used for calculations.

All miRNAs selected based on differential expression were verified as markedly differentially expressed, with $\mathrm{p}$-values varying from $10^{-7}$ to $10^{-21}$ and $\mathrm{FC}$ values up to 95 (Table 3). When comparing HGSC with OSE, 7 and 6 miRNAs were over- and under-expressed in HGSC, respectively. According to FC values, miR-205-5p was the most overexpressed $(F C=74)$, followed by miR-200c-3p, miR-182-5p, miR-141-3p and miR-200b-3p. When comparing CCC with OSE, 11 and 2 miRNAs were overand underexpressed, respectively, including 8 common with the HGSC vs. OSE analysis. miR-182-5p best distinguished CCC from OSE $(F C=66)$, followed by miR200a-3p, miR-200c-3p, miR-200a-5p and 200b-3p. All these miRNAs were overexpressed, whereas miR-383 was the most underexpressed in both HGSC and CCC.

Twelve miRNAs distinguished CCC from HGSC, all except 1 being overexpressed in CCC. The miRNA with highest $\mathrm{FC}$ values was miR-509-3-5p $(\mathrm{FC}=95)$, followed by miR-509-5p, miR-509-3p, miR-510 and miR-508-5p.

Experimental information annotated from IPA for these specific miRNAs is provided in Table 4. As shown, these miRNAs are active regulators of the expression of several cancer-related mRNAs, including ZEB1, ZEB2, VIM, VEGFA, NTRK3 and SPDEF, and most of the miRNAs are cancer-related. The table also highlights differentially expressed miRNAs (Tables 2 and 3) and mRNAs (FC $> \pm 1.5$ ) identified previously [29] in HGSC vs. OSE, 


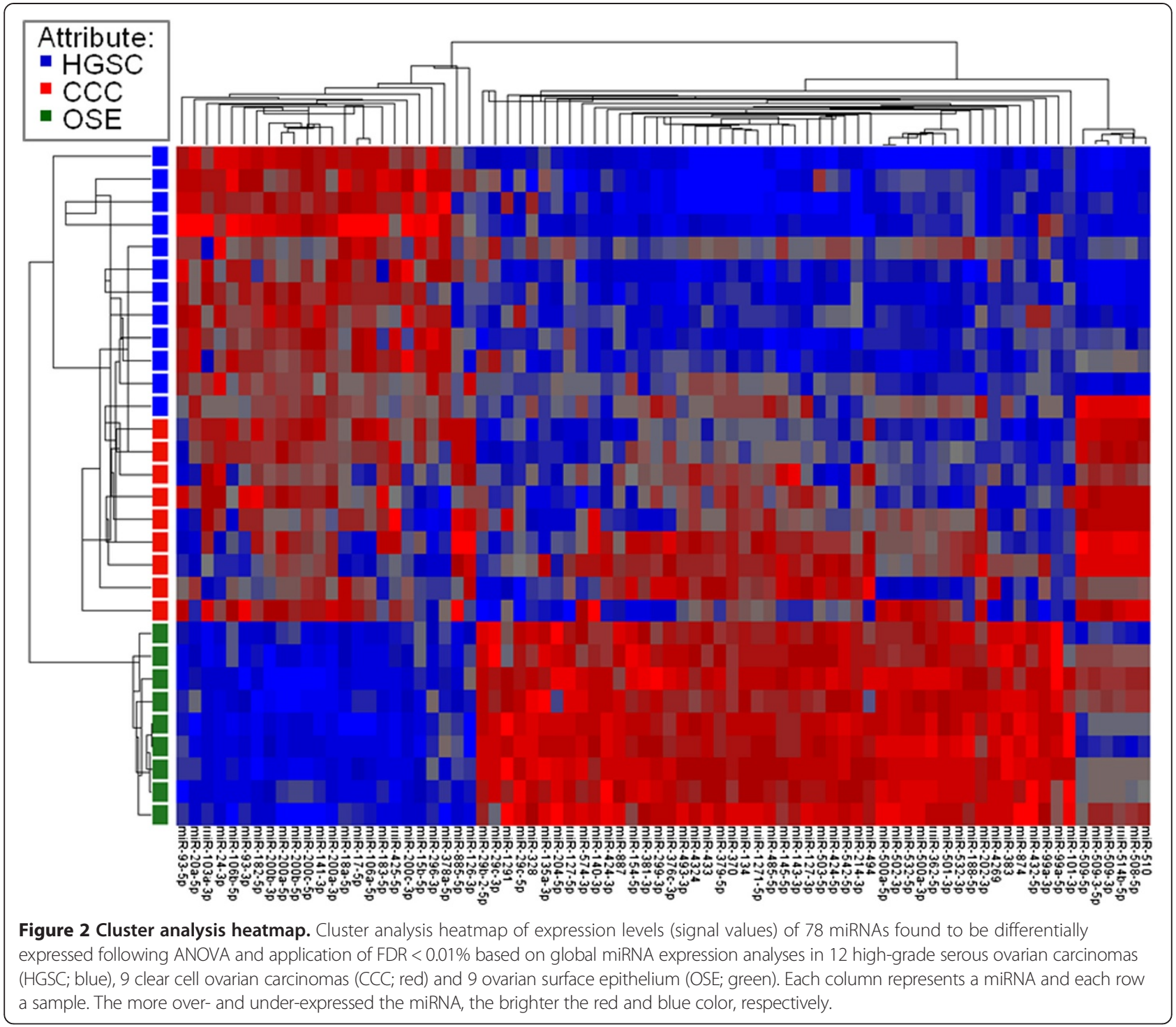

among others ZEB1, ZEB2 and VIM, interacting inversely with miR-200c-3p, miR-200a-3p, miR-205-5p and miR-141-3p. Complete HGSC vs. OSE FC values for the mRNAs listed in Table 4 are provided in Additional file 2 .

\section{Associations between validated miRNA expression and clinical parameters}

All miRNAs validated by RT-qPCR were evaluated for association with PFS, OS, optimal CA125 normalization and residual disease (RD) in patients included in the RT-qPCR analyses. In HGSC, miR-200c-3p was found to be associated with PFS $(p=0.031)$ and $\operatorname{OS}(p=0.026)$. The miR200c-3p FC expression level was divided into tertiles, and Kaplan-Meier plots made (Figure 3). Patients with highest tertile level had shorter OS than patients with intermediate or lowest levels, with median time until death of 18 and
30 months, respectively (Figure 3A). Patients with the highest tertile level had shorter PFS compared with patients with lowest levels, with median time until progression of 7 and 11 months, respectively (Figure 3B). No association was found between the miRNAs and CA 125 normalization or RD (cut-off at $2 \mathrm{~cm}$ ) in HGSC. The 3 miRNAs selected for RT-qPCR based on possible association with survival were not found to be associated with outcome.

In CCC, no associations with PFS or OS were found. However, patients with macroscopic RD (cut-off at $0 \mathrm{~cm})$ had significantly lower miR-202-3p $(\mathrm{p}=0.018)$ and miR-1281 ( $\mathrm{p}=0.035)$ levels $(\mathrm{n}=6$; median $\mathrm{FC}=-5.3$ and -2.0 , respectively) than patients without $\mathrm{RD}(\mathrm{n}=13$; median $\mathrm{FC}=1.6$ and -1.2 , respectively). Associations with CA 125 normalization could not be evaluated in CCC, since all but 3 patients achieved optimal CA 125 normalization. 
Table 2 Differentially expressed miRNAs between HGSC, CCC and OSE of global miRNA expression profiling* selected for RT-qPCR validation

\begin{tabular}{|c|c|c|c|c|c|c|}
\hline \multirow[b]{2}{*}{ miRNAs } & \multicolumn{2}{|c|}{ HGSC vs. OSE ${ }^{a}$} & \multicolumn{2}{|c|}{ CCC vs. OSE ${ }^{a}$} & \multicolumn{2}{|c|}{ CCC vs. HGSC $^{a}$} \\
\hline & p-values & FC values & p-value & FC value & p-value & FC value \\
\hline miR-134 & $8.3 \times 10^{-8}$ & $-16.7^{b}$ & & & $1.0 \times 10^{-4}$ & 5.8 \\
\hline miR-141-3p & $1.1 \times 10^{-11}$ & 46.1 & $2.4 \times 10^{-10}$ & 34.9 & & \\
\hline miR-182-5p & $6.0 \times 10^{-9}$ & 30.2 & $1.4 \times 10^{-8}$ & 32.7 & & \\
\hline miR-200a-3p & $7.3 \times 10^{-10}$ & 33.6 & $1.3 \times 10^{-9}$ & 38.8 & & \\
\hline miR-200a-5p & $3.0 \times 10^{-13}$ & 33.5 & $6.9 \times 10^{-12}$ & 26.5 & & \\
\hline miR-200b-3p & $1.1 \times 10^{-9}$ & 29.1 & $2.9 \times 10^{-8}$ & 21.1 & & \\
\hline miR-200c-3p & $1.2 \times 10^{-12}$ & 16.5 & $1.2 \times 10^{-11}$ & 15.0 & & \\
\hline miR-202-3p & $8.0 \times 10^{-6}$ & -36.9 & & & $2.3 \times 10^{-4}$ & 16.3 \\
\hline miR-205-5p & $4.9 \times 10^{-5}$ & 105.1 & & & $3.1 \times 10^{-3}$ & -23.1 \\
\hline miR-383 & $8.2 \times 10^{-12}$ & -33.7 & $1.5 \times 10^{-11}$ & -38.7 & & \\
\hline miR-424-5p & $2.6 \times 10^{-9}$ & -26.0 & $4.0 \times 10^{-6}$ & -10.1 & & \\
\hline miR-508-5p & & & $4.4 \times 10^{-3}$ & 11.6 & $3.1 \times 10^{-6}$ & 75.0 \\
\hline miR-509-3p & $4.3 \times 10^{-3}$ & -10.3 & & & $2.6 \times 10^{-6}$ & 83.4 \\
\hline miR-509-5p & & & $5.6 \times 10^{-4}$ & 11.4 & $1.8 \times 10^{-6}$ & 34.0 \\
\hline miR-509-3-5p & $3.9 \times 10^{-3}$ & -10.2 & & & $1.9 \times 10^{-6}$ & 84.6 \\
\hline miR-510 & $9.3 \times 10^{-3}$ & -5.2 & $7.9 \times 10^{-3}$ & 6.1 & $3.0 \times 10^{-6}$ & 31.7 \\
\hline miR-513a-5p & & & $4.8 \times 10^{-3}$ & 7.4 & $4.1 \times 10^{-6}$ & 33.5 \\
\hline miR-514b-5p & $9.7 \times 10^{-3}$ & -6.5 & $3.8 \times 10^{-3}$ & 9.8 & $1.3 \times 10^{-6}$ & 63.6 \\
\hline
\end{tabular}

*Affymetrix GeneChip miRNA 2.0 Arrays. ${ }^{a} \mathrm{HGSC}$ : High-grade serous ovarian carcinoma. OSE: ovarian surface epithelium. CCC: Clear cell ovarian carcinoma.

$b_{I_{-}}$illustrates underexpression. FC: Fold change. P-values are calculated on original data (before FDR corrections).

Table 3 Differentially expressed miRNAs $(p<0.01)$ between HGSC, CCC and OSE verified by RT-qPCR

\begin{tabular}{|c|c|c|c|c|c|c|}
\hline \multirow[b]{2}{*}{ miRNAs } & \multicolumn{2}{|c|}{ HGSC vs. OSE $^{a}$} & \multicolumn{2}{|c|}{ CCC vs. OSE ${ }^{a}$} & \multicolumn{2}{|c|}{$\mathrm{CCC}$ vs. $\mathrm{HGSC}^{a}$} \\
\hline & p-values & FC values & p-values & FC values & $p$-values & FC values \\
\hline miR-134 & $8.7 \times 10^{-11}$ & $-5.7^{b}$ & & & $3.1 \times 10^{-6}$ & 4.3 \\
\hline miR-141-3p & $1.7 \times 10^{-18}$ & 40.3 & $7.2 \times 10^{-11}$ & 45.3 & & \\
\hline miR-182-5p & $9.5 \times 10^{-15}$ & 42.4 & $1.2 \times 10^{-8}$ & 66.2 & & \\
\hline miR-200a-3p & $3.6 \times 10^{-5}$ & 33.0 & $9.3 \times 10^{-10}$ & 57.8 & & \\
\hline miR-200a-5p & $3.1 \times 10^{-15}$ & 33.8 & $4.3 \times 10^{-11}$ & 53.0 & & \\
\hline miR-200b-3p & $5.3 \times 10^{-18}$ & 38.8 & $3.7 \times 10^{-11}$ & 51.0 & & \\
\hline miR-200c-3p & $6.0 \times 10^{-21}$ & 48.2 & $3.2 \times 10^{-12}$ & 53.4 & & \\
\hline miR-202-3p & $1.3 \times 10^{-14}$ & -14.7 & & & $1.6 \times 10^{-7}$ & 10.1 \\
\hline miR-205-5p & $9.0 \times 10^{-9}$ & 74.3 & & & $4.4 \times 10^{-3}$ & -8.4 \\
\hline miR-383 & $2.2 \times 10^{-14}$ & -36.6 & $9.8 \times 10^{-10}$ & -15.1 & $2.2 \times 10^{-3}$ & 2.4 \\
\hline miR-424-5p & $3.1 \times 10^{-13}$ & -10.7 & $3.5 \times 10^{-4}$ & -4.2 & $1.6 \times 10^{-3}$ & 2.5 \\
\hline miR-508-5p & & & $3.5 \times 10^{-3}$ & 10.1 & $1.0 \times 10^{-8}$ & 27.5 \\
\hline miR-509-3p & & & & & $2.0 \times 10^{-7}$ & 46.3 \\
\hline miR-509-5p & $5.0 \times 10^{-3}$ & -4.1 & $2.4 \times 10^{-3}$ & 13.3 & $1.3 \times 10^{-8}$ & 54.7 \\
\hline miR-509-3-5p & $1.1 \times 10^{-4}$ & -11.0 & & & $2.2 \times 10^{-8}$ & 95.3 \\
\hline miR-510 & & & $2.5 \times 10^{-3}$ & 9.0 & $8.7 \times 10^{-10}$ & 32.9 \\
\hline miR-513a-5p & & & $6.6 \times 10^{-4}$ & 6.2 & $9.1 \times 10^{-7}$ & 8.3 \\
\hline miR-514b-5p & & & $9.7 \times 10^{-5}$ & 12.1 & $2.3 \times 10^{-9}$ & 25.8 \\
\hline
\end{tabular}

${ }^{a}$ HGSC: High-grade serous ovarian carcinoma. OSE: ovarian surface epithelium. CCC: Clear cell ovarian carcinoma. ${ }^{b_{i}{ }^{\prime}}$ illustrates underexpression. FC: Fold change. 
Table 4 IPA based experimentally observed information for differentially expressed miRNAs and differential expression (FC $> \pm 1.5$ ) of their regulated mRNA targets in HGSC

\begin{tabular}{|c|c|c|c|}
\hline miRNAs & mRNA targets & $\begin{array}{c}\text { Cancer } \\
\text { association }\end{array}$ & $\begin{array}{c}\mathrm{OC} \\
\text { association }\end{array}$ \\
\hline miR-134 $\downarrow$ & & $x$ & \\
\hline miR-141-3p $\uparrow$ & $\begin{array}{l}\text { TGFB2 } \downarrow \text {, ZEB2 } \downarrow \text {, JAG1, BAP1, CLOCK, ELMO2, ERBB2IP, KLHL20, } \\
\text { MAP2K4, PLCG1, PTPRD, WDR37 }\end{array}$ & $x$ & $x(E C)$ \\
\hline $\operatorname{miR}-182-5 p \uparrow$ & 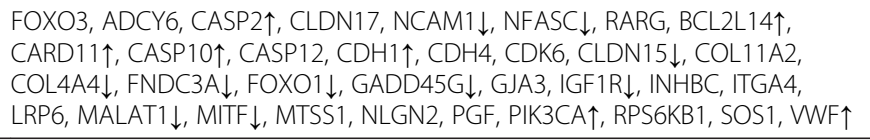 & $x$ & $x(E C)$ \\
\hline$\overline{\operatorname{miR}-200 a-3 p \uparrow}$ & $\begin{array}{l}\text { CTNNB1, VIM } \downarrow \text {, ZEB1 } \downarrow, \text { ZEB2 } \downarrow, \text { BAP1, CDK6, CDKN1B } \downarrow, C T B P 2, C Y P 1 B 1 \uparrow, \\
\text { ELMO2, ERBB2IP, KLHL20, PLCG1, PTPRD } \downarrow, \text { TUBB } \uparrow, W D R 37, \text { ZFPM2 } \downarrow\end{array}$ & $x$ & $x(E C, R O C)$ \\
\hline$\overline{\operatorname{miR}-200 a-5 p \uparrow}$ & & $x$ & \\
\hline $\operatorname{miR}-200 b-3 p \uparrow$ & $\begin{array}{l}\text { VIM } \downarrow, Z E B 1 \downarrow, Z E B 2 \downarrow, \text { BAP1, ELMO2, ERBB2IP, ERRFI1, KLHL20, PLCG1, } \\
\text { PTPRD } \downarrow \text {, RERE, WASF3, WDR37, ZFPM2 } \downarrow\end{array}$ & $x$ & $x(\mathrm{ROC})$ \\
\hline$\overline{m i R-200 c-3 p \uparrow}$ & $\begin{array}{l}\text { CDH1 } \uparrow, \text { PTPN13 } \downarrow, \text { ZEB1 } \downarrow, \text { ZEB2 } \downarrow, \text { FHOD1, PPM1F, JAG1, MARCKS, VIM } \downarrow \text {, } \\
\text { CDKN1B } \downarrow, \text { ERRFI1 } \downarrow, \text { PLCG1 }\end{array}$ & $x$ & $x(E C)$ \\
\hline \multicolumn{4}{|l|}{ miR-202-3p $\downarrow$} \\
\hline miR-205-5p $\uparrow$ & 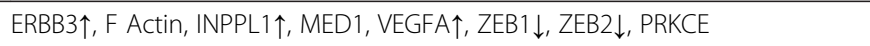 & $x$ & $x(E C)$ \\
\hline \multicolumn{4}{|l|}{ miR-383 $\downarrow$} \\
\hline miR-424-5p $\downarrow$ & FGFR1, MAP2K1, NFIA $\downarrow$, PLAG1 & $x$ & \\
\hline \multicolumn{4}{|l|}{ miR-508-5p } \\
\hline miR-509-3p $\downarrow$ & NTRK3 & & \\
\hline \multicolumn{4}{|l|}{ miR-509-5p $\downarrow$} \\
\hline \multicolumn{4}{|l|}{ miR-509-3-5p $\downarrow$} \\
\hline miR-510 & HTR3E, SPDEF $\uparrow$ & $x$ & \\
\hline miR-513a-5p & CD274 & $x$ & \\
\hline
\end{tabular}

IPA: Ingenuity Pathway Analysis. HGSC: High-grade serous ovarian carcinoma. OC: Ovarian carcinoma. EC: Endometrioid OC. ROC: Recurrent OC. Over- and underexpressed miRNAs (based on Tables 2 and 3) and mRNAs (based on global gene expression analysis [29]) in HGSC vs. ovarian surface epithelium (OSE) are indicated by upward and downward arrows, respectively. A complete list of HGSC vs. OSE FC values for the mRNA targets is available in Additional file 2.

Ingenuity pathway analysis (IPA)

To identify miRNA-mRNA interactions in HGSC, differentially expressed miRNAs in HGSC vs. OSE were linked to differentially expressed mRNAs in HGSC vs. OSE identified previously [29]. miRNAs and mRNAs of the microarray analyses (ANOVA, FDR $<5 \%$ ) were imported to the IPA software and filtered for interactions. When including miRNAs and mRNAs with $\mathrm{FC} \geq \pm 10$, interactions of inverse miRNA-mRNA expression pairing (55.4\% of the interactions), interactions experimentally observed and of high predicted confidence, 19 miRNAs targeting 47 mRNAs (Table 5) were found. All but 3 miRNAs are included in Figure 2. Core analysis was performed, and selected cancer-related functions are shown in Table 5. Fifty-four RNAs were cancer-related, of which 11 mRNAs and 8 miRNAs were OC-related (italics). Thirty-one and 10 molecules were related to cell proliferation and cell cycle, respectively. For a detailed evaluation of the quality of the predicted miRNA-mRNA interactions of Table 5, a plot showing the Context + score as well as number of conserved binding sites of these interactions (TargetScan) is given in Additional file 3 .

We previously presented a HGSC pathway comprising VEGFA, FOXM1, TPX2, BIRC5 and TOP2A, all significantly overexpressed in HGSC vs. OSE and directly interacting with TP53 [29]. Through IPA, these mRNAs were linked to differentially expressed miRNAs in HGSC vs. OSE of the microarray analysis (ANOVA, FDR $<5 \%$, $\mathrm{FC}> \pm 2$ ). When inverse and similar miRNA-mRNA expression pairing and all confidence levels were included, 26 miRNAs and 30 interactions were found (Figure 4). Of these, 7 and 12 were experimentally observed and of high predicted confidence, respectively. Among the miRNAs, 16 were under- and 10 overexpressed. All but 9 miRNAs are included in Figure 2.

\section{Discussion}

In this study, a number of miRNAs distinguishing HGSC and CCC from OSE, as well as CCC from HGSC have been identified, including a set validated by RT-qPCR. 


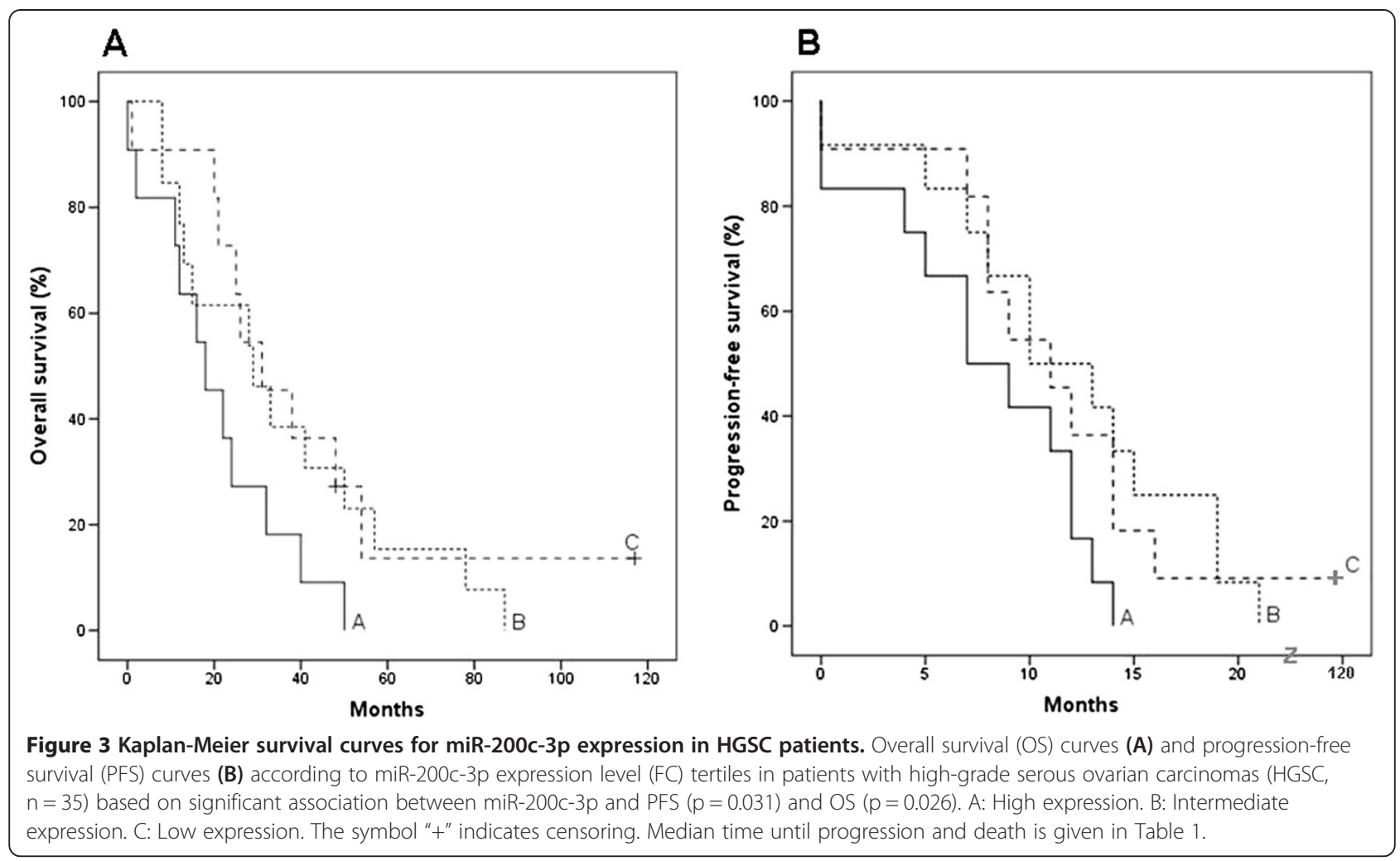

These miRNAs could be involved in the biology of these OC subgroups.

The most differentially expressed miRNAs in both HGSC and CCC compared with OSE were miR-200 family members, including miR-200a-3p, miR-200b-3p, miR-200c-3p and miR-141-3p. These miRNAs are aberrantly expressed in different cancers [33-36], and have been found to be overexpressed in serous and clear cell OC, although few CCC were analyzed $[17,22,26]$.

miR-200 family members have been demonstrated to regulate EMT by targeting ZEB1 and ZEB2, resulting in altered expression of the cell-cell adhesion molecule Ecadherin [37-40]. E-cadherin down-regulation is apparently important in cancer progression, facilitating cell detachment and metastasis. At a favorable distant location, cells may undergo mesenchymal-epithelial transition (MET) and re-express E-cadherin. This is supported by the finding of elevated E-cadherin and reduced ZEB1 in metastatic epithelial ovarian cancer [41], as well as by our findings of overexpressed miR-200 family members and underexpression of ZEB1 and ZEB2 in metastatic HGSC. ZEB1 and ZEB2 are also targets of miR-205-5p [37], which was highly overexpressed in HGSC compared with OSE and CCC.

miR-200c-3p and miR-200b-3p, having similar seed sequences, have been shown to decrease VIM expression and thereby its protein vimentin [39]. Vimentin is found in various non-epithelial cells, especially mesenchymal cells, and is used as marker for EMT during metastasis. Elevated expression of miR-200c-3p and miR-200b-3p, resulting in reduced vimentin levels, is therefore expected in metastatic cancer, where epithelial features are important for re-colonization, in concordance with our findings.

Interestingly, among the IPA documented mRNA targets of the differentially expressed miRNAs in this study, ZEB1 and ZEB2 were among the most underexpressed mRNAs in HGSC compared with OSE. Even though the miRNA-mRNA interactions are not verified in the HGSC material presented, the inverse expression of miR-200 members and ZEB1, ZEB2 and VIM, as well as of miR205-5p and ZEB1 and ZEB2, support a probable interaction also in HGSC.

miR-182-5p had the highest FC in CCC compared with OSE. This miRNA regulates the expression of PIK3CA, a frequently mutated gene in $\mathrm{CCC}$ and a candidate for targeted therapy [42]. Little is known about miR-200a-5p, although it has been related to colorectal cancer [36].

To the best of our knowledge, the present study is the first to identify differentially expressed miRNAs in a relatively large CCC series. The miRNAs most clearly separating CCC from HGSC were miR-509-3-5p and miR-509-5p, having similar seed sequences, as well as miR-509-3p and miR-510. miR-509-3p has been shown to target NTRK3 [43], encoding the receptor tyrosine kinase $\operatorname{TrkC}$, which is involved in the oncogenic PIK3CA 
Table 5 Interacting miRNAs and mRNAs in HGSC differentially* and inversely expressed

\begin{tabular}{|c|c|}
\hline miRNAs and mRNA targets & FC values \\
\hline$m i R-134^{1,5}$ & -16.7 \\
\hline KLHL14 ${ }^{1}$, PAX8 $8^{1,3,5}$ & $9.6-29.3$ \\
\hline miR-141-3p $p^{1,2,6} /$ miR-200a-3p & 46.1 \\
\hline $\mathrm{FOXP2}^{1,2,5}, \mathrm{HLF}^{1}, \mathrm{PCDH} 9^{5}, \mathrm{PEG3}^{1,6}, \mathrm{SCN}^{1} \mathrm{~A}^{1}, \mathrm{SDC}^{1,2,3,4,5,6}$ & $-9.6--24.7$ \\
\hline$m i R-182-5 p^{1,2}$ & 30.2 \\
\hline 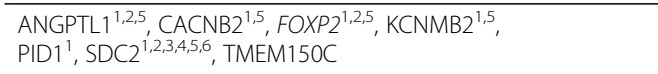 & $-9.6--14.6$ \\
\hline miR-183-5p $1,2,6$ & 11.7 \\
\hline$A B C A 8^{1}, \mathrm{HLF}^{1}$ & $-11.6--17.8$ \\
\hline$m i R-187-3 p^{1}$ & 12.8 \\
\hline TSPAN5 $5^{1,2}$ & -11.3 \\
\hline miR-200b-3p $p^{1,2,6} /$ miR-200c-3p & 29.1 \\
\hline 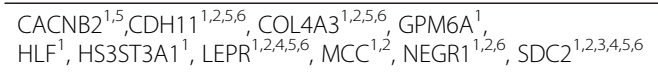 & $-9.6--21.6$ \\
\hline miR-202-3p & -36.9 \\
\hline$R R M 2^{1,2,4,6}$ & 16.9 \\
\hline$m i R-203-3 p^{1,2,6}$ & 13.6 \\
\hline 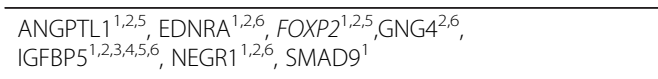 & $-9.7--15.0$ \\
\hline miR-205-5 $p^{1,6}$ & 105.1 \\
\hline $\mathrm{BAMBI}^{1,2,5,6}, \mathrm{NR} \mathrm{C} 2^{1,2,5,6}, \mathrm{PEG} 3^{1,6}$ & $-10.1--24.7$ \\
\hline miR-376c-3p 1 & -11.7 \\
\hline $\mathrm{EHF}^{1,2,3,6}, \operatorname{LRP}^{1,5,6}$ & $11.9-12.6$ \\
\hline miR-379-5p & -10.3 \\
\hline KLHL14 & 29.3 \\
\hline miR-381-3p ${ }^{1}$ & -12.6 \\
\hline $\mathrm{EGFL6}^{1}, \mathrm{NOTCH}^{1,2,3,5,6}$, RRM2 $^{1,2,4,6}$ & $9.7-16.9$ \\
\hline miR-383 & -33.7 \\
\hline MAL2 & 32.8 \\
\hline$m i R-424-5 p^{1,2,3,4,5,6}$ & -26.0 \\
\hline 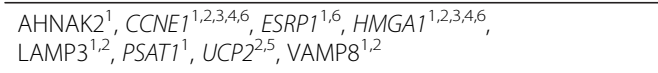 & $9.9-24.0$ \\
\hline miR-485-5p & -13.6 \\
\hline $\mathrm{KRT7}^{1,3,4}, \mathrm{LRP}^{1,5,6}, \mathrm{ST} 14^{1}$ & $10.5-16.2$ \\
\hline miR-887 & -9.6 \\
\hline TMEM139 & 11.7 \\
\hline miR-4324 & -12.5 \\
\hline ERBBB3 $3^{1,2,3,5,6}$, GALNT6 & $17.9-23.7$ \\
\hline
\end{tabular}

HGSC: High-grade serous ovarian carcinoma. *ANOVA, FDR $<5 \%, F C \geq \pm 10$ in HGSC vs. ovarian surface epithelium. All interactions are of high predicted confidence, whereas the interactions between miR-424-5p and CCNE1, HMGA1, PSAT1 and UCP2 are experimentally observed. ${ }^{1,2,3,4,5,6}$ Related to cancer (italicized for ovarian cancer), cellular growth and proliferation, cell cycle, DNA replication, recombination and repair, cell-to-cell signaling and interaction, cellular development, respectively. Results were generated through the use of Ingenuity Pathway Analysis.

pathway. miR-509-3p, miR-509-3-5p and also miR-513a$5 p$ have been found overexpressed in stage I OC [23], and miR-509-5p have been found to inhibit cancer cell proliferation [44]. miR-510 targets SPDEF [45], which have been found underexpressed in OC compared with breast carcinoma [46]. Our findings of an underexpressed miR-
510 and overexpression of SPDEF in HGSC support an interaction also in this cancer subgroup.

In spite of the relatively small sample size, high level of miR-200c-3p was found to be associated with short PFS and OS in HGSC, indicating it may be a prognostic marker for HGSC. This finding is in accordance with a study analyzing miRNA expression in SC vs. normal ovaries [22]. The fact that most of its differentially expressed and experimentally observed mRNA targets were found underexpressed may bolster the conclusion that miR-200c-3p is associated with survival. This miRNA has also been associated with survival in stage I OC patients [47] and chemotherapy response [48]. miR200c-3p was among the most differentially expressed miRNAs in both HGSC and CCC compared with OSE separately, and had the lowest p-value in both comparisons. miR-200c-3p has previously been found to be overexpressed in SC [22,26], HGSC cell lines [49], serum from HGSC patients [49] and in a small series of CCC [26]. Based on the relatively small number of HGSC patients, the findings of the survival analysis should be verified in an extended material, and negative findings should be interpreted with caution.

A larger cohort is warranted for CCC to explore the associations between miRNAs and survival. However, miR-202-3p and miR-1281 were found to be associated with RD in CCC, although this could not be adjusted for stage due to the small series.

We further mapped IPA based interactions between differentially expressed mRNAs and miRNAs in HGSC. Unfortunately, global mRNA expression analysis of CCC was not available. The vast majority of these interacting RNAs has previously been associated with cancer and cancer-related functions, and may represent important key molecular pathways in HGSC. Moreover, differentially expressed miRNAs in HGSC were linked to overexpressed mRNAs in a molecular pathway for HGSC. In this latter IPA analysis, both over- and underexpressed miRNAs were included. The functional association between an overexpressed miRNA and overexpressed miRNA targets, if any, may be indirect or be due to compensatory mechanisms. For example, VEGFA, which we previously found to be overexpressed and associated with PFS in HGSC [29], is a target of miR-200c-3p. A possible explanation for interaction, in spite of both being overexpressed, may be due to adaptive mechanisms leading to overexpression of miR-200c-3p, in an attempt to reduce VEGFA and consequently carcinogenesis. However, an interaction resulting in activation of VEGFA expression can not be ruled out [50-52]. The identified interactions are IPA based, and should be experimentally evaluated in HGSC.

OSE was in this study used as control material, since $\mathrm{OC}$ is presumed to originate in the OSE [53]. However, 


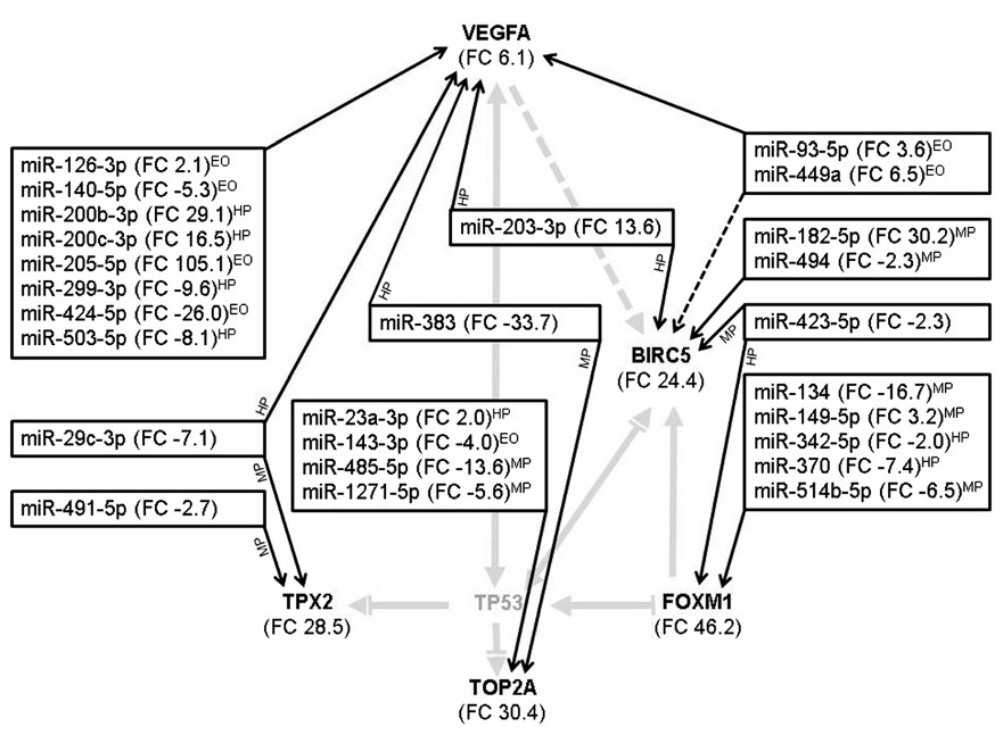

Figure 4 Differentially expressed (FC $\geq 2$ ) miRNAs in HGSC targeting a HGSC molecular pathway of differentially expressed mRNAs [29]. Calculated differential expression is between high-grade serous ovarian carcinomas (HGSC) and ovarian surface epithelium, for miRNAs based on global gene expression analysis (ANOVA, FDR < 5) and for mRNAs based on RT-qPCR analyses [29]. $\rightarrow$ acts on ( - direct interaction, -- indirect interaction), $\perp$ inhibits. FC: Fold change. EO: Experimentally observed interactions. HP: Interactions of high predicted confidence. MP: Interactions of moderate predicted confidence. The abbreviations of the miRNA-mRNA interactions are generally placed after the miRNA FC values, but are placed on the arrow of the miRNA-mRNA interaction for miRNAs having several targets with different interaction type. Results were generated through Ingenuity Pathway Analysis.

an alternative origin of a subset of $\mathrm{OC}$ has recently been proposed, suggesting implanted epithelial cells of the fallopian tube and endometrium in the ovary as an origin for HGSC and CCC, respectively [3]. The basis for this proposed model are findings of tubal dysplasia and tubal intraepithelial carcinoma (TIC) in women predisposed to $[54,55]$ or operated for [56] HGSC, as well as a molecular resemblance of TIC to HGSC [57-59]. However, since a direct transition from lesions in the Fallopian tube to OC has still not been demonstrated, OSE may still be the origin for OC. Interestingly, a common embryological origin of fimbrial epithelium and OSE has been hypothesized [60], which may explain a similar predisposition for the development of tubal and ovarian cancer.

\section{Conclusions}

Several miRNAs significantly differentially expressed between HGSC, CCC and OSE were identified through global miRNA expression profiling and RT-qPCR validation analysis, suggesting a role for these miRNAs in OC. The differences emphasize the biological distinctiveness of these OC subgroups. Highly overexpressed miRNAs including miR-205-5p in HGSC and members of the miR200 family in HGSC and CCC target EMT drivers, and may be important in OC progression. Overexpression of miR-182-5p and miR-200a-5p and underexpression of miR-383 was also found in HGSC and CCC. Some miRNAs separating CCC from HGSC were also identified, including miR-509-3-5p, miR-509-5p, miR-509-3p and miR-510. miR-200c-3p, the most significantly differentially expressed miRNA in both HGSC and CCC, was found to be associated with PFS and OS in HGSC, representing a potential prognostic marker for HGSC. In HGSC, several interacting differentially expressed miRNAs and mRNAs were mapped, but need to be experimentally verified. The identified miRNAs should be explored in future studies as candidate biomarkers and therapeutic targets.

\section{Additional files}

\section{Additional file 1: FC- and p-values for 78 differentially expressed (ANOVA, FDR < $0.01 \%$ ) miRNAs between HGSC, CCC and OSE. \\ Additional file 2: FC values for IPA based experimentally observed mRNA targets of differentially expressed miRNAs in HGSC vs. OSE. \\ Additional file 3: Context + scores for predicted interactions of differentially expressed ( $F C \geq \pm 10$ ) miRNAs and mRNAs in HGSC. miRNAs and predicted mRNA targets are shown in columns. Number of conserved binding sites is given after each mRNA (superscript). All predicted interactions are of high predicted confidence. FC values are provided in Table 5.}

\section{Competing interests}

The authors declare that they have no competing interests.

\section{Authors' contributions}

BVE conceived and designed the experiments, performed patient recruitment, tissue sampling and collection of clinical data, performed experiments and the Ingenuity pathway analyses, analyzed the data, performed statistical analyses and wrote the paper. OKO designed the experiments, performed experiments, analyzed the data and performed statistical analyses. KBFH designed the experiments and analyzed the data. 
BB performed experiments and analyzed the data. LS performed statistical analyses. AS established and was responsible for the research biobank that provided most patient recruitment. KMG designed the experiments and analyzed the data. BD designed the experiments, performed tissue sampling, reviewed the histological material, was consultant in pathology and analyzed the data. All authors discussed the results, contributed to preparation of the manuscript and approved the final manuscript version.

\section{Acknowledgments}

This work was funded by Inger and John Fredriksen Foundation for Ovarian Cancer Research, who had no role in study design, data collection and analysis, decision to publish, or preparation of the manuscript. Professor Claes Trope, OUH, is gratefully acknowledged for valuable clinical advice. We also acknowledge PhD Marit Holden, Chief Research Scientist, Norwegian Computing Center, for statistical support and Lise Levy, technician, OUH, for biobank work and patient recruitment.

\section{Author details}

'Department of Gynecological Oncology, Oslo University Hospital (OUH), The Norwegian Radium Hospital, Postbox 4953 Nydalen 0424, Oslo, Norway. ${ }^{2}$ Department of Medical Biochemistry, OUH, Ullevaal, Oslo, Norway. ${ }^{3}$ Faculty of Medicine, University of Oslo, Oslo, Norway. ${ }^{4}$ Department of Biostatistics and Epidemiology, OUH, Ullevaal, Oslo, Norway. ${ }^{5}$ Department of Gynecology and Obstetrics, OUH, Ullevaal, Oslo, Norway. ${ }^{6}$ Department of Pathology, OUH, The Norwegian Radium Hospital, Oslo, Norway.

Received: 9 August 2013 Accepted: 7 February 2014

Published: 11 February 2014

\section{References}

1. Cancer Registry of Norway: Cancer in Norway 2010 - Cancer incidence, mortality, survival and prevalence in Norway. Oslo: Cancer Registry of Norway; 2012

2. Siegel R, Naishadham D, Jemal A: Cancer statistics, 2012. CA Cancer J Clin 2012, 62:10-29.

3. Prat J: Ovarian carcinomas: five distinct diseases with different origins, genetic alterations, and clinicopathological features. Virchows Arch 2012, 460:237-249.

4. Anglesio MS, Carey MS, Kobel M, Mackay H, Huntsman DG: Clear cell carcinoma of the ovary: a report from the first ovarian clear cell symposium, June 24th, 2010. Gynecol Oncol 2011, 121:407-415.

5. Vang R, Shih I, Kurman RJ: Ovarian low-grade and high-grade serous carcinoma: pathogenesis, clinicopathologic and molecular biologic features, and diagnostic problems. Adv Anat Pathol 2009, 16:267-282.

6. Shih I, Kurman RJ: Ovarian tumorigenesis: a proposed model based on morphological and molecular genetic analysis. Am J Pathol 2004, 164:1511-1518.

7. Huntzinger E, Izaurralde E: Gene silencing by microRNAs: contributions of translational repression and mRNA decay. Nat Rev Genet 2011, 12:99-110.

8. Lu J, Getz G, Miska EA, Alvarez-Saavedra E, Lamb J, Peck D, Sweet-Cordero A, Ebert BL, Mak RH, Ferrando AA, Downing JR, Jacks T, Horvitz HR, Golub TR: MicroRNA expression profiles classify human cancers. Nature 2005, 435:834-838.

9. Torres A, Torres K, Maciejewski R, Harvey WH: MicroRNAs and their role in gynecological tumors. Med Res Rev 2011, 31:895-923.

10. Volinia S, Calin GA, Liu CG, Ambs S, Cimmino A, Petrocca F, Visone R, lorio M, Roldo C, Ferracin M, Prueitt RL, Yanaihara N, Lanza G, Scarpa A, Vecchione A, Negrini M, Harris CC, Croce CM: A microRNA expression signature of human solid tumors defines cancer gene targets. Proc Natl Acad Sci U S A 2006, 103:2257-2261.

11. Kuhlmann JD, Rasch J, Wimberger P: Kasimir-Bauer S: microRNA and the pathogenesis of ovarian cancer-a new horizon for molecular diagnostics and treatment? Clin Chem Lab Med 2012, 50:601-615.

12. Dahiya N, Morin PJ: MicroRNAs in ovarian carcinomas. Endocr Relat Cancer 2010, 17:F77-F89.

13. Vaksman O, Stavnes HT, Kaern J, Trope CG, Davidson B, Reich R: miRNA profiling along tumour progression in ovarian carcinoma. J Cell Mol Med 2011, 15:1593-1602.

14. Li SD, Zhang JR, Wang YQ, Wan XP: The role of microRNAs in ovarian cancer initiation and progression. J Cell Mol Med 2010, 14:2240-2249.
15. Dahiya N, Sherman-Baust CA, Wang TL, Davidson B, Shih I, Zhang Y, Wood W III, Becker KG, Morin PJ: MicroRNA expression and identification of putative miRNA targets in ovarian cancer. PLoS One 2008, 3:e2436.

16. Zhang L, Volinia S, Bonome T, Calin GA, Greshock J, Yang N, Liu CG, Giannakakis A, Alexiou P, Hasegawa K, Johnstone CN, Megraw MS, Adams S, Lassus H, Huang J, Kaur S, Liang S, Sethupathy P, Leminen A, Simossis VA, Sandaltzopoulos R, Naomoto Y, Katsaros D, Gimotty PA, DeMichele A, Huang Q, Butzow R, Rustgi AK, Weber BL, Birrer MJ, et al: Genomic and epigenetic alterations deregulate microRNA expression in human epithelial ovarian cancer. Proc Natl Acad Sci U S A 2008, 105:7004-7009.

17. Yang H, Kong W, He L, Zhao JJ, O'Donnell JD, Wang J, Wenham RM, Coppola D, Kruk PA, Nicosia SV, Cheng JQ: MicroRNA expression profiling in human ovarian cancer: miR-214 induces cell survival and cisplatin resistance by targeting PTEN. Cancer Res 2008, 68:425-433.

18. Shih KK, Qin LX, Tanner EJ, Zhou Q, Bisogna M, Dao F, Olvera N, Viale A, Barakat RR, Levine DA: A microRNA survival signature (MiSS) for advanced ovarian cancer. Gynecol Oncol 2011, 121:444-450.

19. Kim TH, Kim YK, Kwon Y, Heo JH, Kang H, Kim G, An HJ: Deregulation of miR-519a, 153, and 485-5p and its clinicopathological relevance in ovarian epithelial tumours. Histopathology 2010, 57:734-743.

20. Wurz K, Garcia RL, Goff BA, Mitchell PS, Lee JH, Tewari M, Swisher EM: MiR-221 and MiR-222 alterations in sporadic ovarian carcinoma: relationship to CDKN1B, CDKNIC and overall survival. Genes Chromosomes Cancer 2010, 49:577-584.

21. Hu X, Macdonald DM, Huettner PC, Feng Z, El Naqa IM, Schwarz JK, Mutch DG, Grigsby PW, Powell SN, Wang X: A miR-200 microRNA cluster as prognostic marker in advanced ovarian cancer. Gynecol Oncol 2009, 114:457-464.

22. Nam EJ, Yoon H, Kim SW, Kim H, Kim YT, Kim JH, Kim JW, Kim S: MicroRNA expression profiles in serous ovarian carcinoma. Clin Cancer Res 2008, 14:2690-2695.

23. Eitan R, Kushnir M, Lithwick-Yanai G, David MB, Hoshen M, Glezerman M, Hod M, Sabah G, Rosenwald S, Levavi H: Tumor microRNA expression patterns associated with resistance to platinum based chemotherapy and survival in ovarian cancer patients. Gynecol Oncol 2009, 114:253-259.

24. Yang N, Kaur S, Volinia S, Greshock J, Lassus H, Hasegawa K, Liang S, Leminen A, Deng S, Smith L, Johnstone CN, Chen XM, Liu CG, Huang Q, Katsaros D, Calin GA, Weber BL, Butzow R, Croce CM, Coukos G, Zhang L: MicroRNA microarray identifies Let-7i as a novel biomarker and therapeutic target in human epithelial ovarian cancer. Cancer Res 2008, 68:10307-10314.

25. Boren T, Xiong Y, Hakam A, Wenham R, Apte S, Chan G, Kamath SG, Chen DT, Dressman H, Lancaster JM: MicroRNAs and their target messenger RNAs associated with ovarian cancer response to chemotherapy. Gynecol Oncol 2009, 113:249-255.

26. Iorio MV, Visone R, Di LG, Donati V, Petrocca F, Casalini P, Taccioli C, Volinia S, Liu CG, Alder H, Calin GA, Menard S, Croce CM: MicroRNA signatures in human ovarian cancer. Cancer Res 2007, 67:8699-8707.

27. Elgaaen BV, Haug KB, Wang J, Olstad OK, Fortunati D, Onsrud M, Staff AC, Sauer T, Gautvik KM: POLD2 and KSP37 (FGFBP2) correlate strongly with histology, stage and outcome in ovarian carcinomas. PLoS One 2010, 5:e13837.

28. Zorn KK, Jazaeri AA, Awtrey CS, Gardner GJ, Mok SC, Boyd J, Birrer MJ: Choice of normal ovarian control influences determination of differentially expressed genes in ovarian cancer expression profiling studies. Clin Cancer Res 2003, 9:4811-4818.

29. Elgaaen BV, Olstad OK, Sandvik L, Odegaard E, Sauer T, Staff AC, Gautvik KM: ZNF385B and VEGFA are strongly differentially expressed in serous ovarian carcinomas and correlate with survival. PLOS One 2012, 7:e46317.

30. Edgar R, Domrachev M, Lash AE: Gene expression omnibus: NCBI gene expression and hybridization array data repository. Nucleic Acids Res 2002, 30:207-210

31. Livak KJ, Schmittgen TD: Analysis of relative gene expression data using real-time quantitative PCR and the 2(-Delta Delta $C(T)$ ) Method. Methods 2001, 25:402-408.

32. Schmittgen TD, Livak KJ: Analyzing real-time PCR data by the comparative C(T) method. Nat Protoc 2008, 3:1101-1108.

33. Elson-Schwab I, Lorentzen A, Marshall CJ: MicroRNA-200 family members differentially regulate morphological plasticity and mode of melanoma cell invasion. PLoS One 2010, 5. doi: 10.1371/journal.pone.0013176. 
34. Volinia S, Galasso M, Sana ME, Wise TF, Palatini J, Huebner K, Croce CM: Breast cancer signatures for invasiveness and prognosis defined by deep sequencing of microRNA. Proc Natl Acad Sci U S A 2012, 109:3024-3029.

35. Slaby O, Svoboda M, Michalek J, Vyzula R: MicroRNAs in colorectal cancer: translation of molecular biology into clinical application. Mol Cancer 2009, 8:102.

36. Paranjape T, Slack FJ, Weidhaas JB: MicroRNAs: tools for cancer diagnostics. Gut 2009, 58:1546-1554.

37. Gregory PA, Bert AG, Paterson EL, Barry SC, Tsykin A, Farshid G, Vadas MA, Khew-Goodall Y, Goodall GJ: The miR-200 family and miR-205 regulate epithelial to mesenchymal transition by targeting ZEB1 and SIP1. Nat Cell Biol 2008, 10:593-601.

38. Hurteau GJ, Carlson JA, Roos E, Brock GJ: Stable expression of miR-200c alone is sufficient to regulate TCF8 (ZEB1) and restore E-cadherin expression. Cell Cycle 2009, 8:2064-2069.

39. Li Y, VandenBoom TG, Kong D, Wang Z, Ali S, Philip PA, Sarkar FH: Up-regulation of miR-200 and let-7 by natural agents leads to the reversal of epithelial-to-mesenchymal transition in gemcitabine-resistant pancreatic cancer cells. Cancer Res 2009, 69:6704-6712.

40. Kent OA, Mullendore M, Wentzel EA, Lopez-Romero P, Tan AC, Alvarez H, West $\mathrm{K}$, Ochs MF, Hidalgo M, Arking DE, Maitra A, Mendell JT: A resource for analysis of microRNA expression and function in pancreatic ductal adenocarcinoma cells. Cancer Biol Ther 2009, 8:2013-2024.

41. Davidson B, Trope CG, Reich R: Epithelial-mesenchymal transition in ovarian carcinoma. Front Oncol 2012, 2:33.

42. Huynh C, Segura MF, Gaziel-Sovran A, Menendez S, Darvishian F, Chiriboga L, Levin B, Meruelo D, Osman I, Zavadil J, Marcusson EG, Hernando E: Efficient in vivo microRNA targeting of liver metastasis. Oncogene 2011, 30:1481-1488.

43. Muinos-Gimeno M, Guidi M, Kagerbauer B, Martin-Santos R, Navines R, Alonso P, Menchon JM, Gratacos M, Estivill X, Espinosa-Parrilla Y: Allele variants in functional MicroRNA target sites of the neurotrophin-3 receptor gene (NTRK3) as susceptibility factors for anxiety disorders. Hum Mutat 2009, 30:1062-1071.

44. Hidaka H, Seki N, Yoshino H, Yamasaki T, Yamada Y, Nohata N, Fuse M, Nakagawa M, Enokida H: Tumor suppressive microRNA-1285 regulates novel molecular targets: aberrant expression and functional significance in renal cell carcinoma. Oncotarget 2012, 3:44-57.

45. Findlay VJ, Turner DP, Moussa O, Watson DK: MicroRNA-mediated inhibition of prostate-derived Ets factor messenger RNA translation affects prostate-derived Ets factor regulatory networks in human breast cancer. Cancer Res 2008, 68:8499-8506.

46. Stavnes HT, Nymoen DA, Langerod A, Holth A, Borresen Dale AL, Davidson B: AZGP1 and SPDEF mRNA expression differentiates breast carcinoma from ovarian serous carcinoma. Virchows Arch 2013, 462:163-173.

47. Marchini S, Cavalieri D, Fruscio R, Calura E, Garavaglia D, Nerini IF, Mangioni C, Cattoretti G, Clivio L, Beltrame L, Katsaros D, Scarampi L, Menato G, Perego P, Chiorino G, Buda A, Romualdi C, D'Incalci M: Association between miR-200c and the survival of patients with stage I epithelial ovarian cancer: a retrospective study of two independent tumour tissue collections. Lancet Oncol 2011, 12:273-285.

48. Leskela S, Leandro-Garcia $\sqcup$, Mendiola M, Barriuso J, Inglada-Perez L, Munoz I, Martinez-Delgado B, Redondo A, de Santiago J, Robledo M, Hardisson D, Rodriguez-Antona C: The miR-200 family controls beta-tubulin III expression and is associated with paclitaxel-based treatment response and progressionfree survival in ovarian cancer patients. Endocr Relat Cancer 2011, 18:85-95.

49. Kan CW, Hahn MA, Gard GB, Maidens J, Huh JY, Marsh DJ, Howell VM: Elevated levels of circulating microRNA-200 family members correlate with serous epithelial ovarian cancer. BMC Cancer 2012, 12:627.

50. Vasudevan S, Tong Y, Steitz JA: Switching from repression to activation: microRNAs can up-regulate translation. Science 2007, 318:1931-1934

51. Mortensen RD, Serra M, Steitz JA, Vasudevan S: Posttranscriptional activation of gene expression in Xenopus laevis oocytes by microRNAprotein complexes (microRNPs). Proc Natl Acad Sci U S A 2011, 108:8281-8286.

52. Vasudevan S: Posttranscriptional upregulation by microRNAs. Wiley Interdiscip Rev RNA 2012, 3:311-330.

53. Auersperg N, Wong AS, Choi KC, Kang SK, Leung PC: Ovarian surface epithelium: biology, endocrinology, and pathology. Endocr Rev 2001, 22:255-288

54. Piek JM, van Diest PJ, Zweemer RP, Jansen JW, Poort-Keesom RJ, Menko FH, Gille JJ, Jongsma AP, Pals G, Kenemans P, Verheijen RH: Dysplastic changes in prophylactically removed Fallopian tubes of women predisposed to developing ovarian cancer. J Pathol 2001, 195:451-456.

55. Medeiros F, Muto MG, Lee Y, Elvin JA, Callahan MJ, Feltmate C, Garber JE, Cramer DW, Crum CP: The tubal fimbria is a preferred site for early adenocarcinoma in women with familial ovarian cancer syndrome. Am J Surg Pathol 2006, 30:230-236.

56. Kindelberger DW, Lee Y, Miron A, Hirsch MS, Feltmate C, Medeiros F, Callahan MJ, Garner EO, Gordon RW, Birch C, Berkowitz RS, Muto MG, Crum CP: Intraepithelial carcinoma of the fimbria and pelvic serous carcinoma: evidence for a causal relationship. Am J Surg Pathol 2007, 31:161-169.

57. Lee Y, Miron A, Drapkin R, Nucci MR, Medeiros F, Saleemuddin A, Garber J, Birch C, Mou H, Gordon RW, Cramer DW, McKeon FD, Crum CP:

A candidate precursor to serous carcinoma that originates in the distal fallopian tube. J Pathol 2007, 211:26-35.

58. Jarboe E, Folkins A, Nucci MR, Kindelberger D, Drapkin R, Miron A, Lee $Y$, Crum CP: Serous carcinogenesis in the fallopian tube: a descriptive classification. Int J Gynecol Pathol 2008, 27:1-9.

59. Folkins AK, Jarboe EA, Saleemuddin A, Lee Y, Callahan MJ, Drapkin R, Garber JE, Muto MG, Tworoger S, Crum CP: A candidate precursor to pelvic serous cancer ( $\mathrm{p} 53$ signature) and its prevalence in ovaries and fallopian tubes from women with BRCA mutations. Gynecol Oncol 2008, 109:168-173.

60. Auersperg $\mathrm{N}$ : The origin of ovarian carcinomas: a unifying hypothesis. Int J Gynecol Pathol 2011, 30:12-21.

doi:10.1186/1471-2407-14-80

Cite this article as: Vilming Elgaaen et al:: Global miRNA expression analysis of serous and clear cell ovarian carcinomas identifies differentially expressed miRNAs including miR-200c-3p as a prognostic marker. BMC Cancer 2014 14:80.

\section{Submit your next manuscript to BioMed Central and take full advantage of:}

- Convenient online submission

- Thorough peer review

- No space constraints or color figure charges

- Immediate publication on acceptance

- Inclusion in PubMed, CAS, Scopus and Google Scholar

- Research which is freely available for redistribution
C) Biomed Central 